
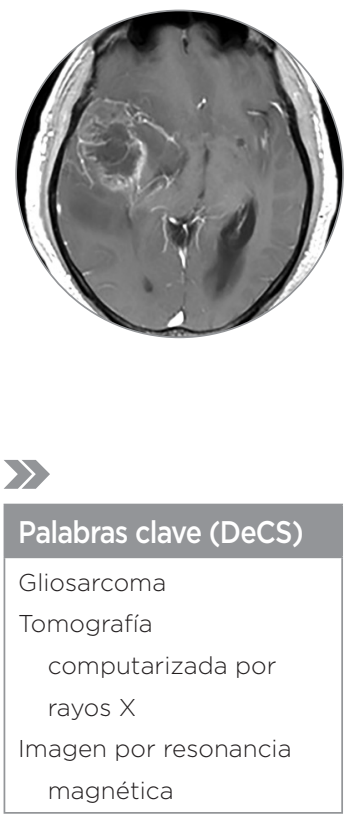

\section{Key words (MeSH)}

Gliosarcoma

Tomography, X-ray computed

Magnetic resonance imaging

\title{
Gliosarcoma: presentación de un caso y hallazgos por tomografía y resonancia magnética convencional y tractografía
}

\author{
Gliosarcoma: Case Report and Findings by Tomography and \\ Conventional and DTI Magnetic Resonance Imaging
}

\author{
Marco Antonio Díaz Escareño ${ }^{1}$ \\ Karla Flores Dávila ${ }^{1}$ \\ Jesús Alexander Cotes Millán ${ }^{1}$ \\ Abril Elena Maciel Fierro ${ }^{2}$ \\ Berenice Zavala Patoni ${ }^{2}$ \\ Katia Hop García ${ }^{3}$ \\ Vicente Arenas Rivera ${ }^{4}$
}

\section{Resumen}

El gliosarcoma es un tumor raro del sistema nervioso central y de alto grado de malignidad. La OMS lo clasifica como variante del glioblastoma (grado IV) y es de mal pronóstico. Histológicamente se caracteriza por tener componentes gliales y mesenquimatosos. El cuadro clínico varía dependiendo de su localización y tamaño, los signos y síntomas más frecuentes son convulsiones, cefalea y déficit neurológico focal. El acercamiento diagnóstico inicial es la tomografía computarizada que aporta datos de sospecha; sin embargo, la resonancia magnética constituye el pilar diagnóstico, con importantes elementos de diagnóstico que se vuelven más significativos con el uso de secuencias funcionales como la tractografía. Se presenta un caso clínico con revisión de la literatura y los hallazgos más significativos en los estudios de imagen.

\section{Summary}

Gliosarcoma is a rare and highly malignant central nervous system tumor. It is classified by the WHO as a variant of glioblastoma (grade IV) and has a poor prognosis. Histologically it is characterized by having both glial and mesenchymal components. Clinically, it varies depending on the location and size of the tumor, the most frequent symptoms being seizures, headaches and focal neurological deficit. The initial diagnostic approach is computed tomography, which provides suspicionus data; however, magnetic resonance is the diagnostic pillar, providing important data that becomes more significant with the use of functional sequences such as tractography. A clinical case is presented with a literature review and the most significant findings in the imaging studies.

\section{Introducción}

El gliosarcoma fue descrito en 1805 por Strobe, es un tumor raro, de alto grado de malignidad y de mal pronóstico.

Es considerado un tumor grado IV por la OMS, sus características principales son: crecimiento rápido, alta tasa mitótica, vasos sanguíneos de neoformación, así como áreas de necrosis. Histológicamente se caracteriza por tener componentes tanto gliales como mesenquimatosos, esto último debido a un estímulo angiogénico que genera hiperplasia microvascular, lo que le confiere la naturaleza bifásica o compuesta $(1,2)$.

Clínicamente, la sintomatología es variable dependiendo de la localización y el tamaño del tumor. Los síntomas más frecuentes son: convulsiones, cefalea, déficit neurológico focal y los relacionados con el aumento de la presión intracraneal.

El acercamiento diagnóstico inicial es la tomografía Hospital de Especialidades del Centro Médico Nacional Siglo XXI. Ciudad de México. constituye el pilar diagnóstico, ya que aporta datos importantes que se vuelven más significativos con el uso de secuencias funcionales como la tractografía que genera información de su relación con los tractos cerebrales; se observa afección de algún tipo en el trayecto de los mismos (3).

\section{Caso clínico}

Paciente masculino de 65 años de edad, con carga genética para diabetes mellitus e hipertensión arterial por parte de los padres. Tabaquismo positivo con índice tabáquico de 18 paquetes-año, hipertensión arterial sistémica de 7 años de evolución en tratamiento médico e hiperplasia prostática obstructiva con tratamiento quirúrgico.

Inició su padecimiento tres meses antes de su ingreso con cuadro caracterizado por cefalea frontal de tipo opresivo de intensidad moderada, $5 / 10$ en escala visual analógica (EVA) que remite espontáneamente, con progresión a cefalea incapacitante asociada a náusea, 
por lo que fue llevado en dos ocasiones a su hospital general de zona y recibió tratamiento sintomático, sin mejoría.

Dos días previos a su ingreso presentó cuadro de paresia y parestesias del hemicuerpo izquierdo.

Fue valorado por el servicio de neurocirugía quienes en la exploración neurológica encontraron funciones mentales conservadas, con lateralización de la marcha hacia la derecha y disminución de la fuerza muscular del hemicuerpo izquierdo, por lo que se solicitó TC de cráneo como abordaje diagnóstico inicial (figura 1).

Ante los hallazgos se realiza RM (figura 2) como complemento diagnóstico y para planeación quirúrgica con secuencia adicional de tractografía (figura 3).

Se decide tratamiento quirúrgico con resección completa extensa y se envía pieza quirúrgica al servicio de anatomía patológica con resultado final de gliosarcoma grado IV según la OMS (figura 4).

El paciente cursa con evolución favorable y se le da el alta.

Se le practicó RM de control (figura 5) 47 días después de la cirugía, que mostró remanente tumoral significativo.

\section{Discusión}

Los tumores del sistema nervioso central representan el $2 \%$ de todas las neoplasias. Constituyen un grupo heterogéneo de neoplasias que incluyen desde lesiones bien diferenciadas y benignas, hasta las altamente invasivas y poco diferenciadas, como el glioblastoma. Recientemente, investigadores del Atlas del Genoma del Cáncer (TCGA por sus siglas en inglés de The Cancer Genome Atlas) establecieron la existencia de cuatro subtipos de glioblastoma (1).

La Organización Mundial de la Salud, en su clasificación de tumores del sistema nervioso central, integra al gliosarcoma como una variante del glioblastoma, grado IV (alto grado de malignidad). Las características de los tumores de este grado son: crecimiento rápido, alta tasa mitótica con vasos de neoformación y áreas de necrosis, lo que le confiere mal pronóstico (2).

Son tumores raros, representan del 2 al $8 \%$ de los glioblastomas y alrededor del $0,48 \%$ de todos los tumores intracraneales.

La historia natural, presentación clínica y el perfil radiológico son similares a los del glioblastoma primario.

Debido a su carácter infiltrante, recidivan hasta en un $90 \%$.

Dentro de los aspectos histológicos que definen al gliosarcoma, se reconoce un bimorfismo de elementos gliales y mesenquimatosos. Las áreas gliales suelen corresponder a glioblastoma y muy raramente puede tener zonas de diferenciación escamosa o glandular con patrones trabecular, adenoide e incluso estructuras papilares. En cuanto al componente mesenquimatoso, este es fenotípicamente el de un fibrosarcoma (3).

Los síntomas ocasionados por un tumor primario del sistema nervioso central se dividen en dos grandes grupos: síntomas focales y síntomas generalizados. Los más frecuentes en la presentación de un tumor de rápido crecimiento como el gliosarcoma, son cefalea, náusea o vómito, así como crisis convulsivas.

El gliosarcoma es un tumor frecuentemente localizado en el lóbulo temporal, por con una mayor relación en el cuadro clínico, otras localizaciones son los lóbulos frontal, parietal y occipital (4).

El abordaje diagnóstico, después de una sospecha clínica, requiere evaluación forzosa con un estudio de imagen. Los estudios de neuroimagen proporcionan información importante que reducen las posibilidades diagnósticas y señalan hacia una etiología probable, aunque el diagnóstico definitivo lo da el estudio histopatológico. Otro tema importante en relación con los estudios de imagen, es la utilidad para la planeación quirúrgica (5).

La TC es útil como abordaje diagnóstico inicial; generalmente se identifica la lesión, su localización, su morfología y evalúa si existe infiltración ósea. Es de especial ayuda cuando se necesita de urgencia la obtención de la imagen o cuando el paciente tiene contraindicación para la RM.

La RM se compone de distintas secuencias que proporcionan información capaz de reducir las opciones diagnósticas, y es el estudio de elección para un tumor del sistema nervioso central.

Generalmente, los gliomas de alto grado se muestran típicamente con baja señal en secuencias ponderadas T1 y alta señal en las ponderadas en $\mathrm{T} 2$, realzan heterogéneamente y de predominio periférico con el medio de contraste paramagnético; producen efecto de masa y edema perilesional importante; suelen tener restricción a la difusión por la alta celularidad que poseen (6).

La espectroscopia se ha usado en los últimos años; permite mejorar la diferenciación de tumores infiltrantes mediante el análisis de los metabolitos del tumor; el N-acetilaspartato (NAA) es un marcador de la integridad celular neuronal que se encuentra disminuido en los gliomas de alto grado, incluido el gliosarcoma; la colina, que es un componente de las membranas celulares, aumenta y el lactato se eleva cuando existe necrosis (7).

La tractografía es una secuencia basada en imágenes del tensor de difusión (DTI) que utiliza el mismo principio de la imagen por difusión. En pacientes con tumores cerebrales es una secuencia fundamental para la distinción de los tractos de sustancia blanca circundantes, y permite distinguir la relación espacial entre el límite del tumor y la sustancia blanca mediante la visualización de las fibras.

Es una secuencia muy útil para la planeación preoperatoria de la resección del tumor, evalúa su relación con fibras relacionadas con funciones cerebrales vitales, como la motora, sensorial, auditiva, visual o del lenguaje. De esta forma se ha convertido en parte integral del diagnóstico preoperatorio en muchos centros neuroquirúrgicos $(8,9)$.

La relación entre los tractos y tumores se clasifica en tres tipos: tipo I o desplazamiento simple, tipo II desplazamiento con interrupción y tipo III interrupción simple; permite la visualización de la relación de los tumores con tractos elocuentes, y en el caso presentado fue beneficiosa en la planeación neuroquirúrgica y la evaluación postoperatoria (8).

Actualmente hay muchas opciones para el tratamiento de neoplasias gliales de alto grado que giran en torno a una combinación de cirugía, radioterapia y agentes quimioterapéuticos.

Las imágenes de RM ponderadas en T1 con medio de contraste son el pilar para el diagnóstico de tumores cerebrales residuales/recurrentes. Son secuencias fáciles de realizar y evalúan con precisión los márgenes de la mayoría de las lesiones tumorales cerebrales tanto primarias como secundarias (10).

La evaluación de la terapia quirúrgica a menudo comienza con una exploración imaginológica para determinar el grado de resección que idealmente deben realizarse dentro de las primeras 24 horas.

Aunque se puede ver un realce reactivo leve después del evento quirúrgico, el realce nodular o similar a la lesión inicial dentro de las 24 horas generalmente representa un tumor residual; sin embargo, distinguir el tumor residual de un cambio reactivo suele ser difícil. 

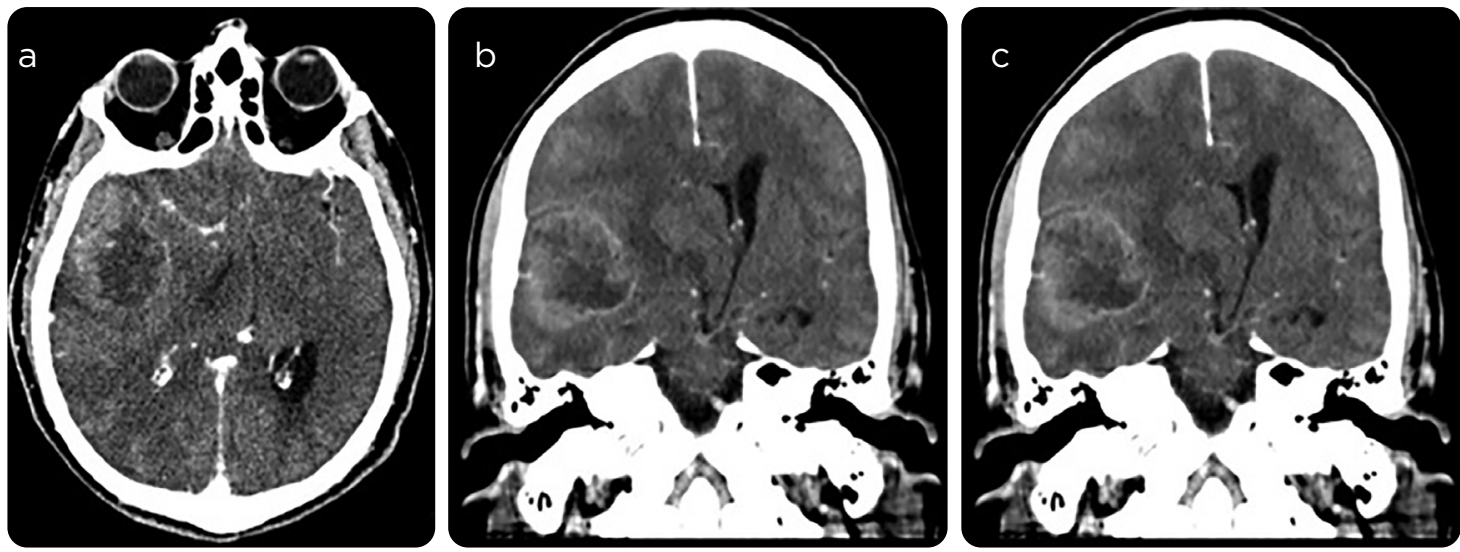

Figura 1. TC de cráneo con medio de contraste. a) Corte axial con reconstrucciones b) coronal y c) sagital. Lesión intraaxial temporal derecha, amorfa, de baja densidad respecto al parénquima cerebral con dimensiones de $35 \times 50 \times 35 \mathrm{~mm}$ en sus ejes longitudinal, anteroposterior y transverso. Muestra realce periférico y efecto de masa sobre el lóbulo frontal, con disminución de la amplitud y profundidad de los surcos y cisuras, el asta frontal y cuerpo del ventrículo lateral, los ganglios basales y el tálamo ipsilaterales, con desviación de la línea media de hasta 15 mm.
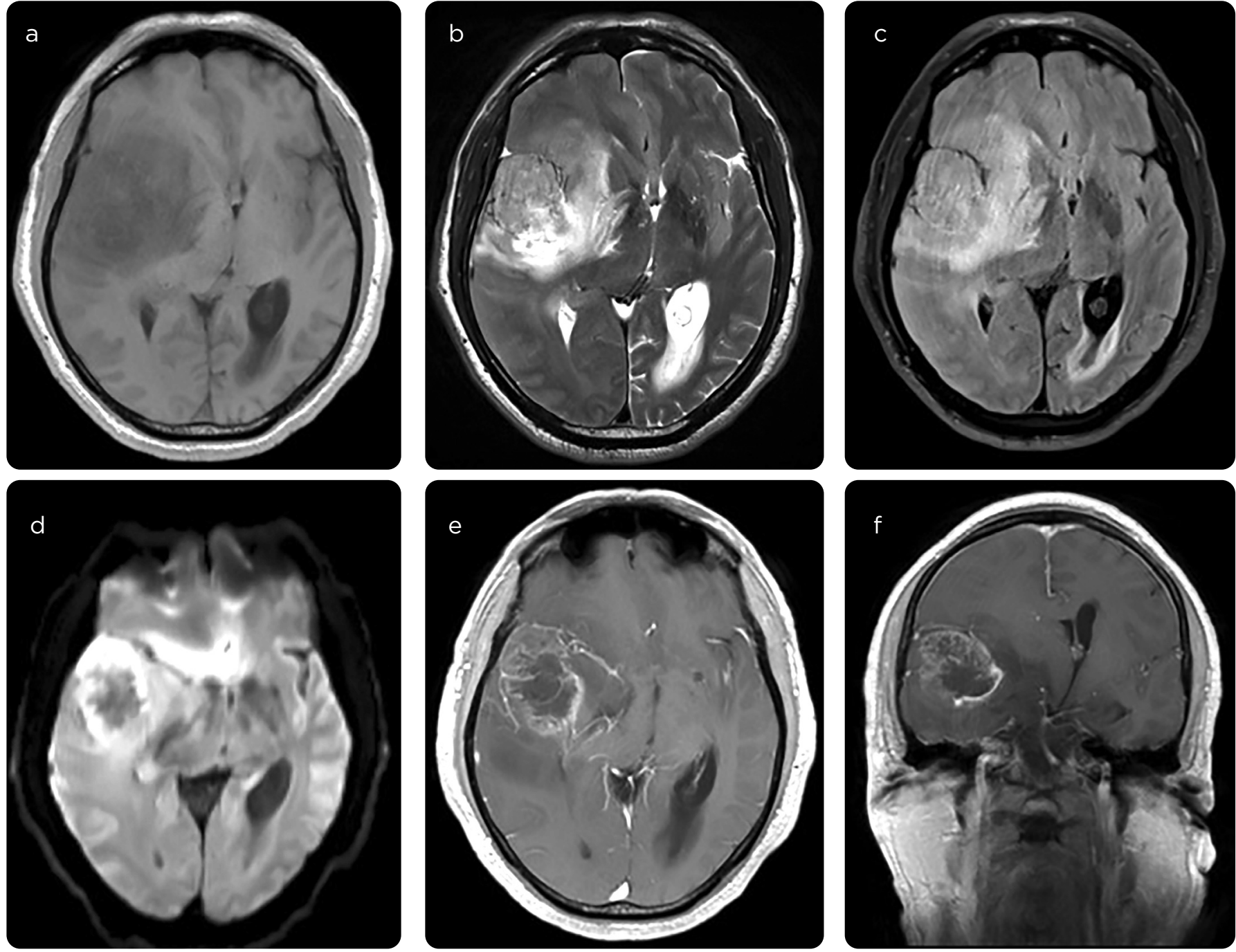

Figura 2. RM de encéfalo convencional. a) Cortes transversales ponderados en T1, b) T2, c) secuencia FLAIR, d) secuencia de difusión, e) secuencia ponderada en T1 con medio de contraste y f) secuencia coronal ponderada en T1 con medio de contraste: lesión supratentorial e intraaxial temporal derecha, ovalada, de $35 \times 50 \times 35 \mathrm{~mm}$ en sus ejes longitudinal, anteroposterior y transverso, heterogénea predominantemente de baja señal en secuencias ponderadas enT1 y de alta señal en las ponderas en T2/FLAIR. En secuencia de difusión: áreas de restricción de localización periférica y tras la administración del medio de contraste realce periférico. Se asocia con importante edema perilesional y efecto de masa sobre el lóbulo frontal asta frontal y cuerpo del ventrículo lateral, los ganglios basales y tálamo, con desviación contralateral de la línea media de hasta $15 \mathrm{~mm}$. 


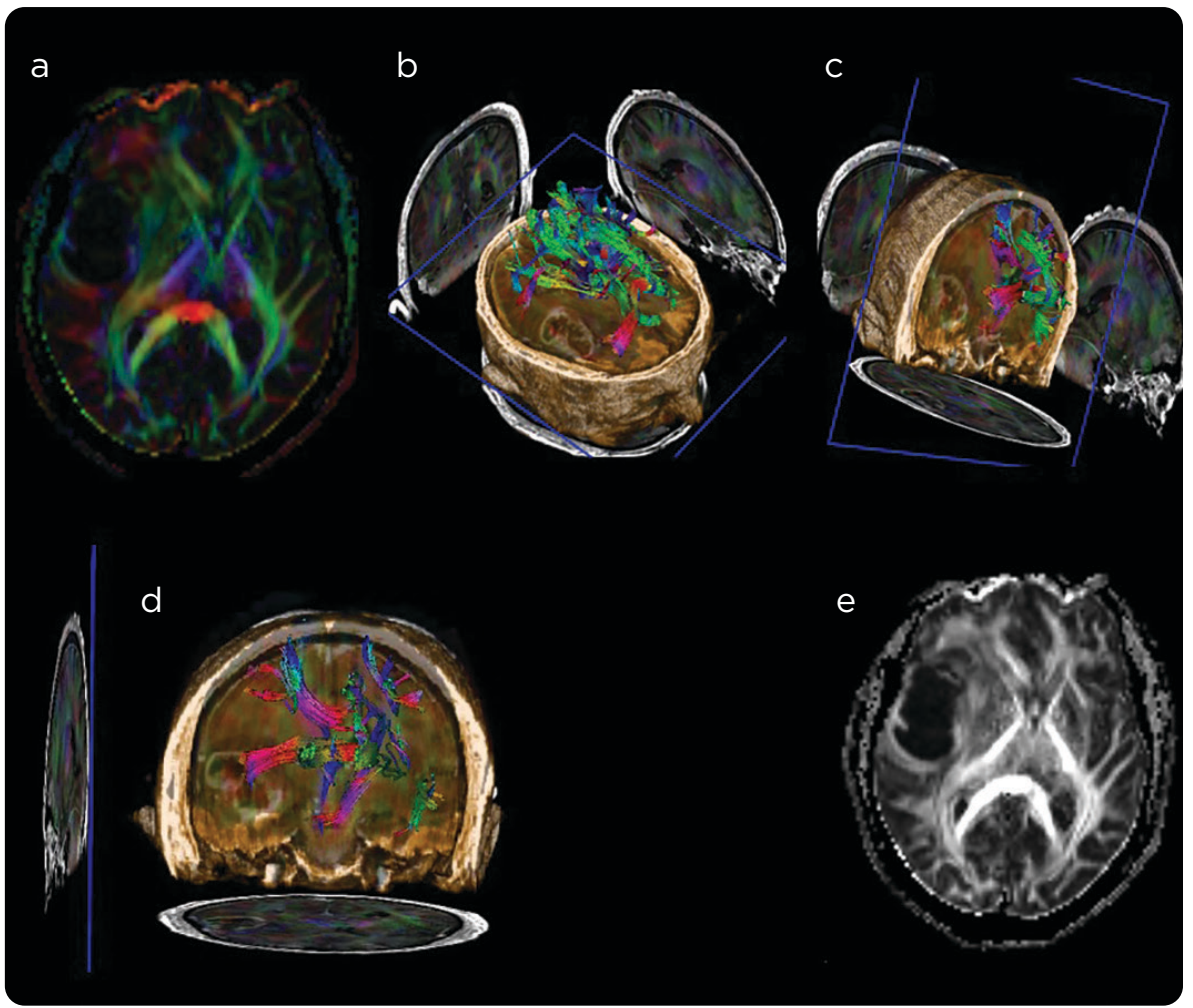

Figura 3. RM de encéfalo con tractografía. a) En corte transversal, b) reconstrucciones volumétricas transversal, y c y d) coronal, e) imagen fuente de tractografía Se observa un compromiso de tipo II: desplazamiento e interrupción de los tractos por lesión.
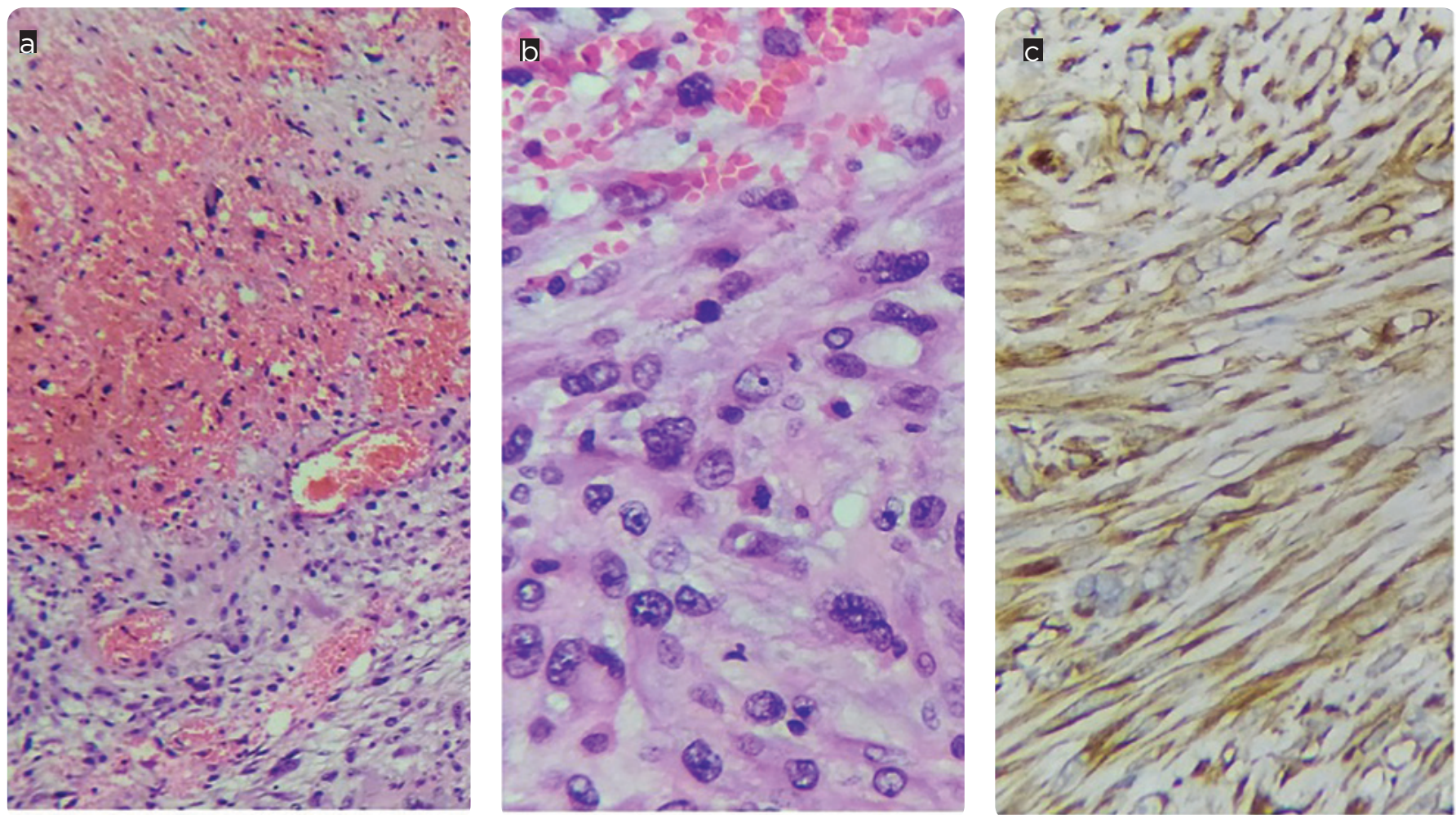

Figura 4. Los cortes histológicos muestran una neoplasia hipercelular con proliferación atípica de astrocitos pleomórficos de aspecto maligno. Hay zonas de necrosis focal, proliferación vascular y actividad mitótica. Algunas áreas simulan un glioblastoma típico; sin embargo, hay células fusiformes y núcleos atípicos. Con la inmunotinción positiva para vimentina, se confirma el componente mesenquimatoso de la neoplasia. 


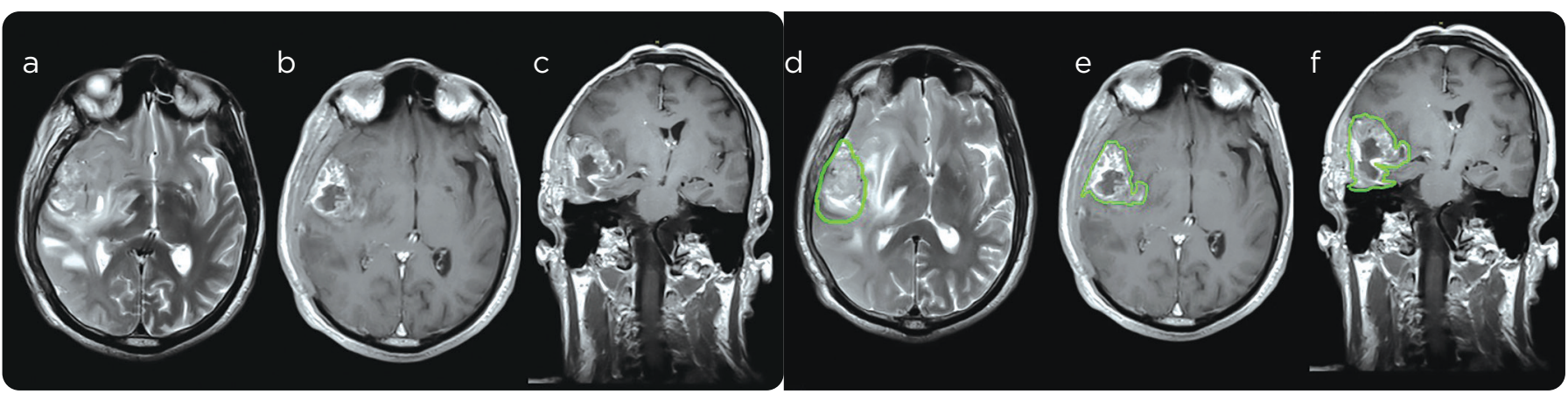

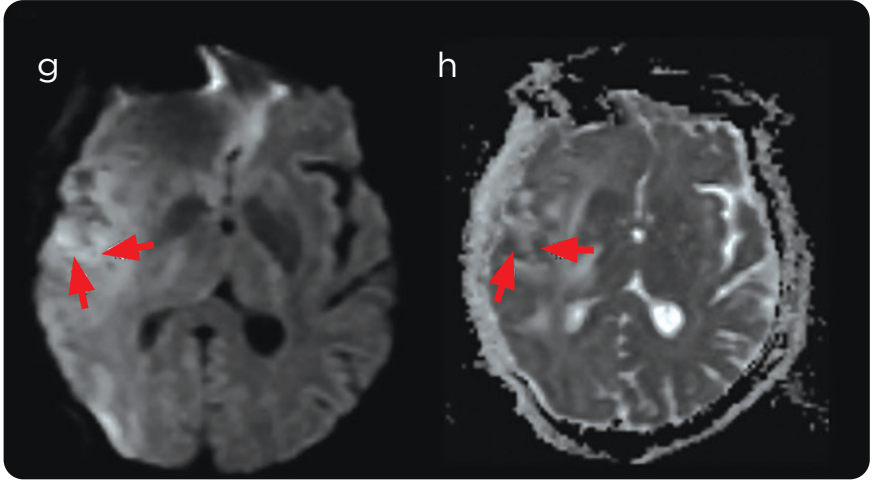

Existen otras secuencias que pueden ayudar al momento de diagnosticar lesión cerebral recurrente. Las imágenes ponderadas en T2/ FLAIR pueden ser útiles para diferenciar entre áreas de tumor residual sin realce y reacción posquirúrgica. Esta secuencia demuestra zonas de intensidad de señal anormal y su extensión, aunque en gliomas de alto grado puede no diferenciar de manera confiable el tumor infiltrante del edema vasogénico, ya que ambos se muestran de alta señal.

Las imágenes ponderadas en difusión aportan información sobre la difusividad de agua. Muchos procesos fisiopatológicos resultan en restricción a la difusión, como ocurre en las neoplasias gliales de alto grado; por lo tanto, es importante correlacionar cualquier nuevo realce con zonas de restricción a la difusión que pudieran corresponder a recurrencia o progresión tumoral.

Otras secuencias útiles para la evaluación de recurrencia o progresión son la perfusión y la espectroscopia.

Individualmente, ninguna de estas ha demostrado ser muy específica; sin embargo, en conjunto, estas secuencias permiten diagnosticar tumor residual y diferenciarlo de cambios secundarios al tratamiento oncológico (11).

\section{Referencias}

1. De Robles P, Fiest KM, Frolkis AD, Pringsheim T, Atta C, St Germaine-Smith C, et al. The worldwide incidence and prevalence of primary brain tumors: a systematic review and meta-analysis. Neuro Oncol. 2015;17(6):776-83.

2. Boerman RH, Anderl K, Herath J, et al. The glial and mesenchymal elements of gliosarcomas share similar genetic alterations. J Neuropathol Exp Neurol. 1996;55:973-81.

3. Kakkar N, Kaur J, Singh G, Singh P, Siraj F, Gupta A. Gliosarcoma in young adults: A rare variant of glioblastoma. W J Oncology. 2017;8(2):53-7.

4. Maiuri F, Stella L, Benvenuti D, et al. Cerebral gliosarcomas: correlation of computed tomographic findings, surgical aspect, pathological features, and prognosis. Neurosurgery. 1990;26:261-7.

5. Anderson MD, Colen RR, Tremont-Lukats IW. Imaging mimics of primary malignant tumors of the central nervous system (CNS). Curr Oncol Rep. 2014;16:399.
Figura 5. RM de encéfalo de control posquirúrgico. a) Cortes transversales ponderados en T2, b) Ponderados en T1 con medio de contraste, c) reconstrucción coronal, d) secuencia difusión y e) mapa de ADC: cambios quirúrgicos por craniectomía pterional derecha. En el lecho quirúrgico en el lóbulo temporal derecho remanente tumoral de morfología irregular, heterogéneo predominantemente hipointenso respecto al parénquima en secuencias ponderadas en T1 e hiperintenso en secuencias ponderadas en T2. Muestra realce periférico, giral y meníngeo reactivo y edema vasogénico asociado a desplazamiento medial de los ganglios de la base con colapso parcial del ventrículo lateral del mismo lado. En secuencia de difusión y mapa de ADC: restricción a la difusión (alta señal en difusión y baja en mapa de ADC) de los bordes de la lesión que muestran realce.

6. Chourmouzi D, Papadopoulou E, Marias K, Drevelegas A. Imaging of brain tumors Surg Oncol Clin N Am. 2014;23(4):629-84.

7. Burtscher IM, Skagerberg G, Geijer B, Englund E, Ståhlberg F, Holtås S. Proton MR spectroscopy and preoperative diagnostic accuracy: an evaluation of intracranial mass lesions characterized by stereotactic biopsy findings. AJNR Am J Neuroradiol. 2000;21:84-93.

8. Ma J, Su S, Yue S, Zhao Y, Li Y, Chen X, Ma H, et al. Preoperative visualization of cranial nerves in skull base tumor surgery using diffusion tensor imaging technology. Turk Neurosurg. 2016;26(6):805-12.

9. WI, Zhang F, Unadkat P, et al. White matter tractography for neurosurgical planning: A topography-based review of the current state of the art. Essayed NeuroImage: Clin. 2017;15:659-72.

10. Wen PY, Macdonald DR, Reardon DA, Cloughesy TF, Sorensen AG, Galanis E, Degroot J, Wick W, Gilbert MR, Lassman AB, Tsien C, Mikkelsen T, Wong ET, Chamberlain MC, Stupp R, Lamborn KR, Vogelbaum MA, van den Bent MJ, Chang $\mathrm{SM}$. Updated response assessment criteria for high-grade gliomas: response assessment in neuro-oncology working group. J Clin Oncol. 2010;28(11):1963-72.

11. Hygino da Cruz LC Jr, Rodríguez I, Dominguez RC, Gasparetto EL, Sorensen AG. Pseudoprogression and pseudoresponse: imaging challenges in the assessment of posttreatment glioma. AJNR Am J Neuroradiol. 2011; 32(11):1978-85.

\section{Correspondencia}

Marco Antonio Díaz Escareño

Av. Cuauhtémoc 330, Doctores, 06725

Ciudad de México, México

mdiazesc@gmail.com

Recibido para evaluación: 20 de noviembre de 2019 Aceptado para publicación: 20 de febrero de 2020 